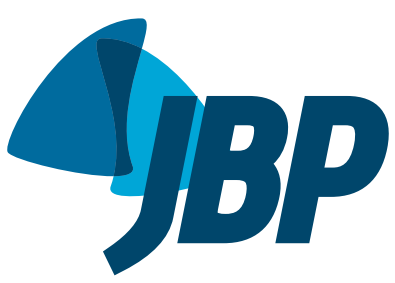

\section{Prominent bronchial vasculature, hemoptysis, and bilateral ground-glass opacities in a young woman with mitral stenosis}

Fabian Aigner $^{1}$, Rudolf Speich ${ }^{1}$, Macé Matthew Schuurmans ${ }^{1}$
A 22-year-old woman presented with hemoptysis and dyspnea (respiratory rate, 38 breaths/min; peripheral oxygen saturation, $81 \%$; pro-brain natriuretic peptide, $2,073 \mathrm{ng} / \mathrm{L})$, together with bilateral inspiratory crackles and a diastolic murmur over the apex. Imaging showed multifocal consolidations surrounded by ground-glass opacities, predominantly in the upper lobes (Figure 1), and no pulmonary embolism. Bronchoscopy showed no endobronchial blood but prominent bronchial vasculature on the left (Figure 2). The BAL fluid was consistently bloody ( $90 \%$ hemosiderin-laden alveolar macrophages). Echocardiography detected severe mitral stenosis with classic "hockey stick" morphology of the anterior leaflet and mild aortic regurgitation. The mean pulmonary artery pressure was $48 \mathrm{mmHg}$, and the pulmonary artery wedge pressure was $32 \mathrm{mmHg}$. Mitral valve reconstruction was performed.

Mitral stenosis can provoke hemoptysis. (1) Dilatation of the bronchial vasculature may be the first hint of elevated left atrial pressure. The bronchial venous plexus arising from the bronchial arterial circulation is connected to the pulmonary venous circulation. Two thirds of the blood from the venous plexus returns to the pulmonary veins and then to the left atrium. $(2,3)$ An increase in pulmonary venous pressure leads to a reverse flow of blood from the pulmonary veins to the bronchial venous plexus, visible as engorged bronchial vasculature.

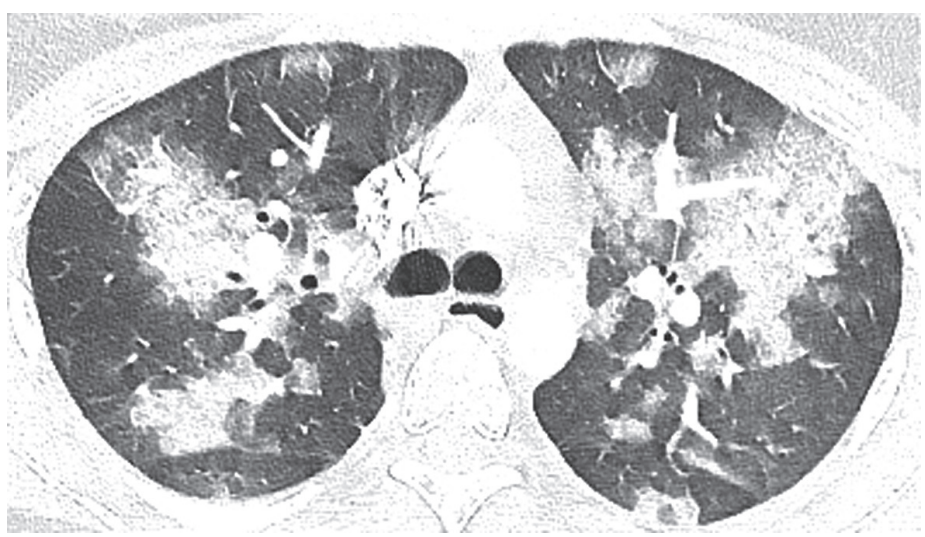

Figure 1. CT of the chest at the level of the main carina showing bilateral consolidations surrounded by ground glass opacities.

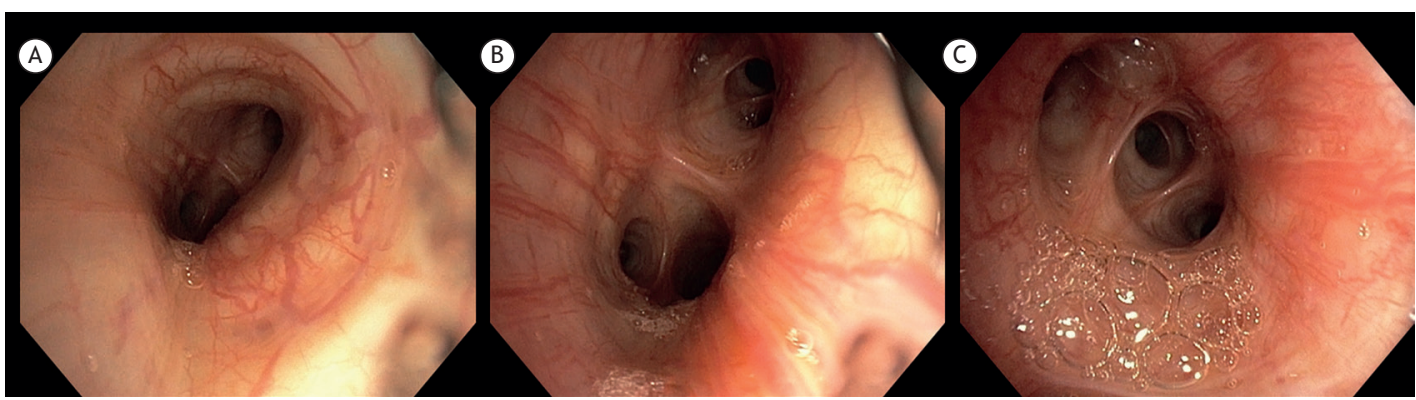

Figure 2. In A, fiberoptic bronchoscopy image of the main left bronchus. In B, a closer view at the left secondary upper lobe carina. In C, a close-up of the left superior bronchus showing the lingula and left upper lobe segments. The bronchial mucosa is edematous and contains a network of prominent blood vessels. The vessels are dilated and engorged to a variable extent. At some sites, the vessels are focally distributed, although most parts of the bronchial mucosa exhibit a dense and partially confluent vascular network.

\title{
RECOMMENDED READING
}

1. Wood P. An appreciation of mitral stenosis. I. Clinical features. Br Med J. 1954;1(4870):1051-63; contd. http://dx.doi.org/10.1136/ bmj.1.4870.1051

2. Ohmichi M, Tagaki S, Nomura N, Tsunematsu K, Suzuki A
Endobronchial changes in chronic pulmonary venous hypertension. Chest. 1988;94(6):1127-32. http://dx.doi.org/10.1378/chest.94.6.1127

3. Baile EM. The anatomy and physiology of the bronchial circulation. J Aerosol Med. 1996;9(1):1-6. http://dx.doi.org/10.1089/jam.1996.9.1 